\title{
Sensitive Method to Identify and Characterize Proteinases In Situ after SDS-PAGE
}

BioTechniques 29:1108-1113 (November 2000)

\section{Jennifer Williams, William J. McGrath and Walter F. Mangel \\ Brookhaven National Laborato- ry, Upton, NY, USA}

\section{INTRODUCTION}

A technique to identify and characterize highly specific proteinases in situ after SDS-PAGE of a crude cell extract should be quite useful. There are techniques for identifying and characterizing relatively nonspecific proteinases in situ after SDS-PAGE (e.g., trypsin-like serine proteinases) $(2-4,12,14,15)$. A gel is cast in the presence of casein. After electrophoresis, the gel is washed with nonionic detergent until the SDS is removed and the proteinase refolds. Then, the reactivated proteinase begins to degrade the casein. If the gel is stained with a dye specific for protein, clear bands will appear where the casein has been degraded and, therefore, where a proteinase activity has migrated. This is an excellent technique for proteinases that are relatively nonspecific. However, most proteinases are more specific than trypsin; they would therefore cleave fewer sites in casein. Furthermore, the amounts of specific proteinases in cells and body fluids are low compared to the amounts of other proteins and nucleic acids. Thus, a very specific and sensitive assay is needed to detect proteinase activity in SDS-polyacrylamide gels after electrophoresis. Here, we present such a method using rhodamine-based fluorogenic substrates.

Previously, we had designed and synthesized an extremely sensitive and specific class of substrates for proteinases (5). The reporter group is rhodamine-
110, a diamino analogue of fluorescein that, like fluorescein, is one of the most detectable compounds known (Figure 1A). In the quinone state, rhodamine 110 exhibits an extremely high molar absorbance coefficient, greater than 70000 $\mathrm{M}^{-1} \mathrm{~cm}^{-1}$, and an extremely high quantum yield, greater than $90 \%$. Its fluorescence intensity is maximal in the high acidic to basic $\mathrm{pH}$ range. It absorbs maximally at $492 \mathrm{~nm}$, where the output from a xenon lamp is relatively high and interference from most biological com pounds is low. When both amino groups on rhodamine 110 are derivatized, for example, with benzyloxycarbonyl-arginine (Cbz-Arg) to give (Cbz-Arg-NH) ${ }_{2}$-rhodamine, the rhodamine moiety shifts into the lactone state in which it does not absorb 492-nm light (Figure 1C). Cleavage of one of the two amide bonds to yield $\mathrm{Cbz}$-Arg-NH-rhodamine results in a conversion of the rhodamine moiety back to the quinone state with a concomitant 3500-fold increase in fluorescence intensity (Figure 1B).

Here, we define the sensitivity of a method to identify and characterize proteinases in situ after SDS-PAGE. The model system is trypsin, and its cleavage is of a rhodamine-based substrate. Although (Cbz-Arg-NH) ${ }_{2}$-rhodamine is an excellent substrate for bovine trypsin, the tri-peptide derivative (Cbz-Ile-Pro-Arg-NH) ${ }_{2}$-rhodamine is even better; its $\mathrm{k}_{\mathrm{cat}} / \mathrm{K}_{\mathrm{m}}$ is $625000 \mathrm{M}^{-}$ $1_{\mathrm{s}^{-1}}$, fourfold greater than that of $(\mathrm{Cbz}-$ Arg-NH) 2 -rhodamine (7). 


\section{MATERIALS AND METHODS}

\section{Sample Preparation and Electrophoresis}

Bovine trypsin $(10 \mathrm{mg} / \mathrm{mL}$ stored at $-20^{\circ} \mathrm{C}$ in $1 \mathrm{mM} \mathrm{HCl}$ ) from Sigma (St. Louis, MO, USA) was serially diluted by factors of 10 into HEPES-buffered saline (25 mM HEPES, pH 7.4, 137 $\mathrm{mM} \mathrm{NaCl}, 2.68 \mathrm{mM} \mathrm{KCl}, 0.5 \mathrm{mM}$ $\mathrm{MgCl}_{2}$ and $0.9 \mathrm{mM} \mathrm{CaCl}_{2}$ ) at $0^{\circ} \mathrm{C}$. To each sample of diluted trypsin, 0.25 volume of $4 \times$ SDS-PAGE sample buffer (80 mM Tris-HCl, pH 8.5, 8\% SDS, 4 mM EDTA and $0.004 \%$ bromophenol blue) was added, and the samples were incubated at $23^{\circ} \mathrm{C}$ for 5 min. Electrophoresis was performed in a PhastSystem ${ }^{\mathrm{TM}}$ (Amersham Pharmacia Biotech $\mathrm{AB}$, Uppsala, Sweden) in precast $8 \%-25 \%$ polyacrylamide gradient PhastGels ${ }^{\circledR}$ (Amersham Pharmacia Biotech) to which $3.5 \mu \mathrm{L}$ sample was applied to each lane.

\section{Renaturation of the Proteinases In Situ}

After electrophoresis, the gel was submerged in $25 \mathrm{mM}$ HEPES ( $\mathrm{pH} 8.0$ ) containing $10 \%$ ethanol for $25 \mathrm{~min}$ at $23^{\circ} \mathrm{C}$. Next, the gel was incubated in 10 mM HEPES, $\mathrm{pH}$ 8.0, $2.5 \%$ Triton $^{\circledR} \mathrm{X}$ 100 and $1 \mathrm{mM}$ EDTA for $30 \mathrm{~min}$ and then in $10 \mathrm{mM}$ HEPES, $\mathrm{pH} 8.0,10 \mathrm{mM}$ $\mathrm{CaCl}_{2}$ and $10 \%$ glycerol for $10 \mathrm{~min}$. Finally, the gel was air-dried in a petri dish for 15 min to allow excess moisture to evaporate.

\section{Preparation of the Gel Overlay}

Meanwhile, substrate-soaked Whatman ${ }^{\circledR}$ No. 1 filter paper (Whatman, Clifton, NJ, USA) was prepared as follows: (Cbz-Ile-Pro-Arg-NH) ${ }_{2}$-rhodamine $(0.01 \mathrm{M}$ in dimethylformamide [DMF]) synthesized as described in Reference 7 was diluted to $100 \mu \mathrm{M}$ in a volume of $0.5 \mathrm{~mL}$ containing $20 \mathrm{mM}$ HEPES, pH 7.4, $15 \%$ ethanol. That solution was then poured on one side of a piece of Whatman No. 1 filter paper cut slightly larger than the gel. After a 15min incubation, excess fluid was evaporated with the aid of a cool hair dryer. Substrate overlays can be prepared in advance if stored at $4^{\circ} \mathrm{C}$.

\section{Visualization of the Fluorescence in Bands}

The reactivated enzyme was located in situ by placing the substrate overlay on top of the slightly moist gel and then pressing them together gently but firm. ly to insure uniform contact. This filter paper/gel sandwich was encased in plastic wrap and incubated at $37^{\circ} \mathrm{C}$ for $2 \mathrm{~h}$. The increase in fluorescence in the bands on the gel with proteinase activity could be followed in real time with a hand-held UV light set at the long wavelength.

\section{RESULTS}

\section{Assay of Five Different Concentrations of Trypsin}

To demonstrate the sensitivity of the method, five amounts of trypsin differing by factors of 10 and ranging in amount from $3.5 \mu \mathrm{g}$ to $350 \mathrm{pg}$ were fractionated by SDS-PAGE. After washing the gel to remove the SDS and to allow the enzyme to refold, a piece of Whatman No. 1 filter paper impregnated with (Cbz-Ile-Pro-Arg-NH) ${ }_{2}-$ rhodamine was placed on top of the gel. Periodically, a hand-held UV light was used to observe the development of the fluorescent bands. The gel was then placed on a transilluminator with a 600-nm cutoff filter (16-18) and photographed with a charge-coupled device (CCD) camera (Figure 2a). Bands of fluorescence were easily observed in the presence of 3500, 350, 35 and 3.5 ng trypsin. The band from $350 \mathrm{pg}$ trypsin was faint, a sixth lane on the gel (data not shown) contained $35 \mathrm{pg}$ trypsin; fluorescence above background could not reproducibly be observed. However, fluorescence from 35 pg trypsin could be observed in gels incubated with the overlay for longer time periods and whose lanes did not contain nanogram or higher concentrations of trypsin (data not shown).

\section{Quantitation of Assay Results}

The fluorescence in the bands can be quantitated. In Figure 2b, lanes of the substrate-soaked Whatman No. 1 filter paper overlay were scanned by a fluorescence imaging system. The first two lanes were purposely overexposed so the sensitivity of the technique could be demonstrated in the other lanes. The overexposure in lanes a and $\mathrm{b}$ are signified by the broad plateau where trypsin migrated in the gel. Lanes c-e show the intensity of the fluorescence due to the hydrolysis of (Cbz-Ile-Pro-Arg-NH) ${ }_{2}-$ rhodamine was somewhat proportional to the amount of trypsin applied to the gel. It appears as if the intensity of fluorescence was directly proportional to the amount of trypsin in lanes c and d; the intensity of fluorescence differed by about tenfold. The intensity of fluorescence in lane e was too close to background fluorescence to appear to be directly proportional to the amount of trypsin added.

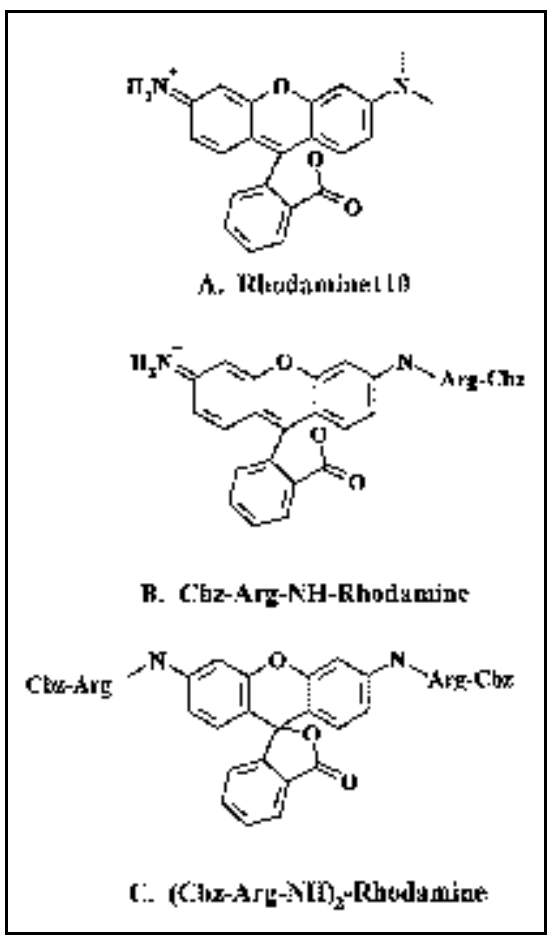

Figure 1. (A) The structure of the reporter group rhodamine110; (B) the structure of the hydrolysis product Cbz-Ile-Pro-Arg-NH-rhodamine; (C) the substrate (Cbz-Ile-Pro-Arg$\mathrm{NH}_{2}$-rhodamine. The structure of rhodamine110 is shown in the quinone state. The structure of the substrate (Cbz-Ile-Pro-Arg-NH) ${ }_{2}$-rhodamine is shown with the rhodamine moiety in the lactone state. The structure of the hydrolysis product Cbz-Ile-Pro-Arg-NH-rhodamine is shown with the rhodamine moiety in the quinone state. Upon hydrolysis of an Arg-NH amide bond in the substrate by trypsin to yield Cbz-Ile-ProArg-NH-rhodamine, there is a 3500-fold increase in fluorescence intensity. 


\section{Research Report}

\section{DISCUSSION}

\section{Rationale for the Different Steps in the Assay}

Certain parts of the assay we described were designed specifically for measuring trypsin activity, and others were not. No reducing agent was used in the SDS-PAGE sample buffer, and the sample was incubated for $5 \mathrm{~min}$ at $23^{\circ} \mathrm{C}$ as opposed to being placed in a boiling water bath. These precautions ensured that the enzyme did not become irreversibly denatured. On the other hand, since it may not be completely denatured during SDS-PAGE, its mobility in the gel may not be absolutely indicative of its molecular weight. Ethanol was present in the first wash to remove the SDS from the protein. Triton X-100 was present in the second wash to displace the remaining SDS and allow trypsin to refold into an active conformation. Different conditions may be necessary with other proteinases. $\mathrm{CaCl}_{2}$ was present in the final wash just before the gel was dried because trypsin requires it for maximal activity. It is at this step that one should use a buffer system optimal for the enzyme to be assayed. Glycerol was present in the final wash because, even though the gel was eventually allowed to air-dry, the glycerol present kept the gel slightly moist. This moisture allows for good contact between the gel and the dry substrate overlay. If there is too much moisture, the bands on the overlay broaden because of diffusion.

For trypsin, a concentration of 100 $\mu \mathrm{M}$ substrate was optimal; below that, only fluorescence from high concentrations of trypsin could be observed; above that, diffusion of the fluorescent product became a problem. The substrate overlay paper should be dry before applying it to the slightly damp gel; otherwise, the product of substrate hydrolysis will diffuse away more readily from the band of proteinase that generated it. Finally, with trypsin, the reaction between substrate and proteinase was limited to $2 \mathrm{~h}$; otherwise, product diffusion leading to band broadening became evident. Detection and quantitation of fluorescence is dependent on the concentration of the fluorophore so that band broadening will

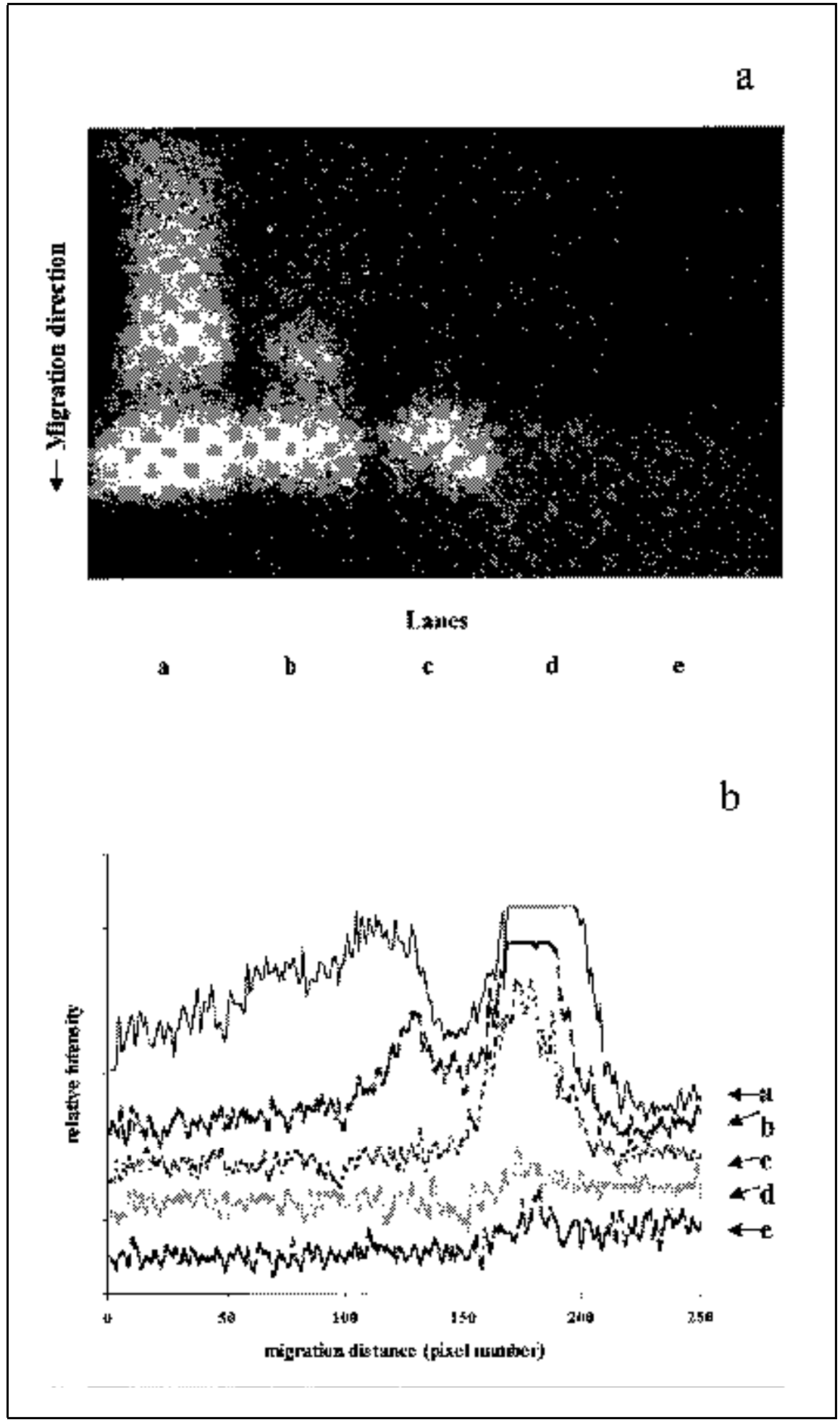

Figure 2. Bovine trypsin activity in situ after SDS-PAGE: (a) location of bovine trypsin activity and (b) quantitation of renatured enzyme activity. (a) Lanes a-e contained $3.5 \mu \mathrm{g}, 350 \mathrm{ng}, 35 \mathrm{ng}, 3.5 \mathrm{ng}$, and $350 \mathrm{pg}$ bovine trypsin, respectively. SDS-PAGE, renaturation of the enzyme and overlay of the gel with Whatman No. 1 filter paper impregnated with (Cbz-Ile-Pro-Arg-NH) $)_{2}$-rhodamine were done as described in the text. In panel a, the overlay was placed on a transilluminator and photographed with a CCD camera containing a 600-nm bandpass filter. In panel b, the overexposed image in panel a was scanned lane by lane. The location of the trypsin band is signified by an arrow. Image intensity analysis was performed using the University of Texas Health Science Center at San Antonio ImageTool program (developed at UTHSCSA and available from the Internet by anonymous FTP from masrad6.uthscsa.edu). 
decrease the sensitivity of detection.

The procedures we used to visualize and quantitate the fluorescent bands can be improved. There was a sixth lane on the gel in Figure 2a (data not shown); it contained $35 \mathrm{pg}$ trypsin. Here, fluorescence could not reproducibly be observed in photographs because the background was variable. However, with a hand-held UV lamp, we could easily see the band corresponding to $35 \mathrm{pg}$ trypsin. The transilluminator and the filters on the CCD camera we used were set up to detect ethidium bromide fluorescence, not fluorescence from rhodamine 110 . Im agers set up for fluorescein wavelengths using argon lasers are commercially available and can detect much lower concentrations of substrate hydrolysis products. To detect very weak signals from bands, the Whatman No. 1 filter paper can be viewed in a fluorescence microscope, especially a confocal microscope, set up to observe fluorescein. If low amounts of proteinase are to be assayed, the adjacent lanes on the gel should contain no sample; this will reduce the background fluorescence and help keep it uniform.

\section{Assay for Proteinase Inhibitors}

Not only can one determine substrate specificity and apparent size of proteinases fractionated by SDSPAGE, but one can also obtain inhibitor profiles. The same sample is run in two adjacent lanes on a gel. After renaturation of the enzymes, the two lanes are separated. One lane is soaked with potential inhibitor, the other with buffer. Then, the same substrate overlay is placed on top of both lanes. The presence of an inhibitor is signified by less fluorescence in the lane with the potential inhibitor compared to that in the lane of the control. If potential inhibitors are proteins, such as bovine pancreatic trypsin inhibitor, they can be incorporated into the polyacrylamide solution before it is polymerized to form a gel. Alternatively, a potential inhibitor, even an antibody, can be incorporated onto Whatman No. 1 filter paper as a substrate, the antibody overlay placed on one side of the gel and the substrate overlay placed on the other side of the gel. Weder et al. (20) de- scribe an in situ gel assay for proteinase inhibitors, where the inhibitor is fractionated by SDS-PAGE, the gel is incubated with proteinase and then a cellulose acetate overlay with a fluorogenic substrate for the proteinase is placed on the gel. The fluorogenic substrates they used were 7-amino-4-methylcoumarinbased analogues. The corresponding rhodamine-based substrates are much more sensitive; they exhibit an increase in sensitivity with most enzymes of 50to 300-fold compared to 7-amino-4methylcoumarin-based analogues (5).

\section{Fluorogenic Substrates with $\mathbf{P}_{\mathbf{1}}-\mathbf{P}_{\mathbf{1}}^{\prime}$ Specificity}

These in situ assays are not restricted to proteinases that recognize only amino acids in a substrate that are $\mathrm{N}$-terminal to the cleavage site. Some proteinases recognize amino acids in substrates that are both $\mathrm{N}$-terminal and $\mathrm{C}$-terminal to the cleavage site. For example, omptin cleaves between basic amino acids. We recently developed an assay for omptin (11) that exploits the observation that aminopeptidases will not cleave $\mathrm{N}$-terminal blocked amino acids such as benzyloxycarbonylarginylarginine 4methyl-7-coumarylamide (Cbz-ArgArg-NH Mec). However, if Cbz-ArgArg-NH Mec is cleaved by omptin to yield Arg-NH Mec, then that product is a substrate for aminopeptidase $\mathrm{M}$, and the product of that reaction will be the highly fluorescent Mec. Thus, to identify and characterize such proteinases in situ in SDS-polyacrylamide gels, an aminopeptidase can be soaked into the overlay along with the substrate.

\section{Highly Selective Rhodamine-Based Substrates}

There is a wide variety of rhodamine-based substrates that can be used in this in situ assay; most are highly specific, and some are highly selective. For example, since trypsin-like serine proteinases cleave after lysine or arginine residues, the substrates $(\mathrm{Cbz}-$ Arg-NH $)_{2}$-rhodamine or $(\operatorname{Arg}-\mathrm{NH})_{2}$ rhodamine can be used to identify all trypsin-like enzymes applied to a gel (5). However, there are also highly specific and selective rhodamine-based substrates. The specificity of a sub- 


\section{Research Report}

strate for an enzyme can be described by the specificity constant, $\mathrm{k}_{\text {cat }} / \mathrm{K}_{\mathrm{m}}$, which reflects the efficiency with which the enzyme catalyses the hydrolysis of the substrate. The selectivity of a substrate refers to whether it is efficiently hydrolyzed by one enzyme and not by others. For example, some of the best rhodamine-based substrates for human plasmin are among the worst substrates for human thrombin, and vice versa. The substrates with the two highest specificity constants with human plasmin, (Cbz-Phe-Arg-NH) ${ }_{2}$-rhodamine and (Cbz-Trp-Arg-NH) $)_{2}$-rhodamine, are not hydrolyzed by human thrombin (6). Conversely, the best substrate for human thrombin, (Cbz-ProArg-NH) ${ }_{2}$-rhodamine, is one of the worst substrates for human plasmin, the difference in $\mathrm{k}_{\text {cat }} / \mathrm{K}_{\mathrm{m}}$ values being 40 fold. The human adenovirus proteinase exhibits extreme selectivity towards substrates. A systematic study of the cleavage of octapeptides by the adenovirus proteinase indicates that the main determinants of proteinase specificity are the amino acids at the $\mathrm{P}_{4}$ position (Leu, Ile or Met), the $\mathrm{P}_{2}$ position (Gly) and the $\mathrm{P}_{1}$ position (Gly) (19). Only rhodamine-based substrates with the following sequences are cleaved by the enzyme: $\mathrm{P}_{4}$ (Leu, Ile, Met), $\mathrm{P}_{3}$ $(\mathrm{Xxx}), \mathrm{P}_{2}$ (Gly), $\mathrm{P}_{1}$ (Gly), where (Xxx) is any amino acid $(1,10,13)$. The adenovirus proteinase could not be assayed with gelatin or casein because these proteins do not contain adenovirus proteinase cleavage sequences.

\section{Advantages of the Assay}

There are several advantages to the assays described here: (i) many different proteinases in an extract can be characterized in one gel lane. Characterization is not limited to the interaction with just one substrate because several overlays each with a different substrate can be used to probe the same gel lane. (ii) Assays can be designed to determine substrate specificity. For example, substrate overlays can contain many different rhodamine-based substrates. Once fluorescence is observed in a mixture, the rhodamine-based substrates in that mixture can be tested individually. (iii) If a band corresponding to an unknown proteinase appears, that enzyme can be purified and characterized using the substrate used to identify it. (iv) For most of the assays described here, pre-cast gels that are commercially available can be used. The exception is for those assays that require a protein to be present in the polyacrylamide solution before it is polymerized to form a gel. (v) Many of the rhodamine-based substrates are available commercially $(8,9)$, and (vi) substrate overlays need not be limited to probe proteinases fractionated by SDS-PAGE. The overlays can be used after nondenaturing PAGE or after isoelectric focusing gel electrophoresis. Rhodamine 110 , as a reporting group, is particularly advantageous in the latter technique because its florescence is insensitive to $\mathrm{pH}$. 


\section{ACKNOWLEDGMENTS}

This research was supported by the Office of Biological and Environmental Research of the U.S. Department of Energy under Prime Contract No. DEAC02-98CH10886 with Brookhaven National laboratory and by National Institute of Allergy and Infectious Diseases Grant No. AI41599. J.W. was supported by the U.S. Department of Energy, Division of University and Industry Programs, Office of Energy Research, as a Science and Engineering Research Semester Program participant. The transilluminator and CCD camera were supported by the U.S. Department of Energy Office of Biological and Environmental Research to Dr. J.C. Sutherland.

\section{REFERENCES}

1.Ding, J., W.J. McGrath, R.M. Sweet and W.F. Mangel. 1996. Crystal structure of the human adenovirus proteinase with its 11 amino-acid cofactor. EMBO J. 15:1778-1783.

2.Foltmann, B., P.B. Szecsi and N.I. Tarasova. 1985. Detection of proteases by clotting of casein after gel electrophoresis. Anal. Biochem. 146:353-360.

3.Heussen, C. and E.G. Dowdle. 1980. Electrophoretic analysis of plasminogen activators in polyacrylamide gels containing sodium dodecyl sulfate and copolymerized substrates. Anal. Biochem. 102:196-202.

4.Kelleher, P.J. and R.L. Juliano. 1984. Detection of proteases in polyacrylamide gels containing covalently bound substrates. Anal. Biochem. 136:470-475.

5.Leytus, S.P., L.L. Melhado and W.F. Mangel. 1983. Rhodamine-based compounds as fluorogenic substrates for serine proteases. Biochem. J. 209:299-307.

6.Leytus, S.P., W.L. Patterson and W.F. Mangel. 1983. New class of sensitive, specific, and selective substrates for serine proteinases: fluorogenic, amino acid peptide derivatives of rhodamine. Biochem. J. 215:253-260.

7.Leytus, S.P., D.L. Toledo and W.F. Mangel. 1984. Theory and experimental method for determining individual kinetic constants for fast-acting, irreversible, protease inhibitors. Biochim. Biophys. Acta 788:74-86.

8.Mangel, W.F., S.P. Leytus and L.L. Melhado. 1985. Rhodamine derivatives as fluorogenic substrates for proteinases. United States patent $4,557,862$.

9.Mangel, W.F., S.P. Leytus and L.L. Melhado. 1987. Novel Rhodamine derivatives as fluorogenic substrates for proteinases. United States Patent 4,640,893.

10.Mangel, W.F., W.J. McGrath, D.L. Toledo and C.W. Anderson. 1993. Viral DNA and a viral peptide can act as cofactors of adenovirus virion proteinase activity. Nature
361:274-275.

11.Mangel, W.F., D.L. Toledo, M.T. Brown, K. Worzalla, M. Lee and J.J. Dunn. 1994. Omptin: an Escherichia coli outer membrane proteinase that activates plasminogen. Methods Enzymol. 244:384-399.

12.Manicourt, D.H. and V. Lefebvre. 1993. An assay for matrix metalloproteinases and other proteases acting on proteoglycans, casein, or gelatin. Anal. Biochem. 215:171-179.

13.McGrath, W.J., A.P. Abola, D.L. Toledo, M.T. Brown and W.F. Mangel. 1996. Characterization of human adenovirus proteinase activity in disrupted virus particles. Virology 217:131-138.

14.Raser, K.J., A. Posner and K.K. Wong. 1995. Casein zymography: a method to study mu-calpain, m-calpain, and their inhibitory agents. Arch. Biochem. Biophys. 319:211216.

15.Roche, P.C. and R.J. Ryan. 1986. Electrophoretic analysis of membrane proteases in the lutenized rat ovary. Endocrinology 119:495-501.

16.Sutherland, J.C. 1993. Electronic imaging of electrophoretic gels and blots, p. 1-42. In A. Chrambach, M.J. Dunn and B.J. Radola (Eds.), Advances in Electrophoresis. VCH Publishers, New York.

17.Sutherland, J.C., B. Lin, D.C. Monteleone, B.M. Sutherland and J. Trunk. 1987. Electronic imaging system for direct and rapid quantitation of fluorescence from electrophoretic gels. Anal. Biochem. 163:446457.

18.Sutherland, J.C., B.M. Sutherland, A. Emrick, D.C. Monteleone, E.A. Ribeiroea, J. Trunk, M. Son, P. Serwer, S.K. Poddar and J. Maniloff. 1991. Quantitative electronic imaging of gel fluorescence with CCD cam eras: applications in molecular biology. BioTechniques 10:492-497.

19. Webster, A., S. Russell, P. Talbot, W.C. Russell and G.D. Kemp. 1989. Characterization of the adenovirus proteinase: substrate specificity. J. Gen. Virol. 70:3225-3234

20.Weder, J.K., K. Haussner and M.V. Bokor. 1993. Use of fluorogenic substrates to visualize trypsin and chymotrypsin inhibitors after electrophoresis. Electrophoresis 14:220-226.

Received 16 February 2000; accepted 5 July 2000.

Address correspondence to:

Walter F. Mangel

Biology Department

Brookhaven National Laboratory

Upton, NY 11973-5000, USA

e-mail:mangel@bnl.gov 